\title{
A Multicentre PDA Project to Support the Clinical Decision Process
}

\author{
ET van der Velde ${ }^{1}$, DE Atsma ${ }^{1}$, R Hoekema ${ }^{2}$, JE Luijten ${ }^{2}$, \\ CI Buddelmeijer ${ }^{3}$, HJ Spruijt ${ }^{4}$, NHJJ van der Putten ${ }^{5}$ \\ ${ }^{1}$ Leiden University Medical Center, Leiden, The Netherlands \\ ${ }^{2}$ University Medical Center Nijmegen, Nijmegen, The Netherlands \\ ${ }^{3}$ Amsterdam Medical Center, Amsterdam, The Netherlands \\ ${ }^{4}$ VU Medical Center, Amsterdam, The Netherlands \\ ${ }^{5}$ Erasmus Medical Center, Rotterdam, The Netherlands
}

\begin{abstract}
In the Netherlands, the departments of Cardiology of all academic hospitals work together under the auspices of the Interuniversity Cardiology Institute of the Netherlands (ICIN). In many of these ICIN centers, PDA's are used to support the cardiologist in his daily practice. In these centers PDA versions are being developed of relevant clinical guidelines. Also, medical PDA applications are evaluated and distributed locally. To give a boost to these local efforts, representatives of 5 ICIN centers have started a PDA collaboration project, with the purpose to jointly develop or find PDA applications to support the clinical decision process.

Relevant cardiology guidelines, such as the European (ESC) and national guidelines have been made available for the PDA. In addition, we have selected PDA applications that will be used in all centers, such as riskprediction software, medical calculators and drug reference software. All PDA clinical guidelines and medical applications have been made available for download on a special website.
\end{abstract}

\section{Introduction}

In the Netherlands, the departments of Cardiology of all University Medical Centers work together under the auspices of the Interuniversity Cardiology Institute of the Netherlands (ICIN). In many of these centers, PDA's (Personal Digital Assistant) (Palm ${ }^{\mathrm{TM}}$, Pocket-PC) are used to support the cardiologist in the medical decision making process. In these ICIN centers, PDA versions of relevant clinical guidelines are being developed. Also various medical PDA applications are evaluated and distributed locally. To give a boost to these local efforts, representatives of a number of ICIN centers have started a PDA collaboration project, with the purpose to jointly develop PDA guidelines and/or select PDA applications to support the clinical decision process. The ICIN representatives are the local ICT experts (at each department of Cardiology), and together they form the 'ICIN-ict projects group'.

\section{Methods}

Relevant cardiology guidelines, such as the European (ESC) and national guidelines have been selected and have been made available for the PDA [1]. Since in the ICIN centers two types of PDA document readers (iSilo ${ }^{\mathrm{TM}}$, http://www.isilo.com and Tomeraider ${ }^{\mathrm{TM}}$, http://www.tomeraider.com) are used, the documents have been made available in both formats. The source documents for the PDA documents are created in HTML format, so only one single source is needed for all of the documents.

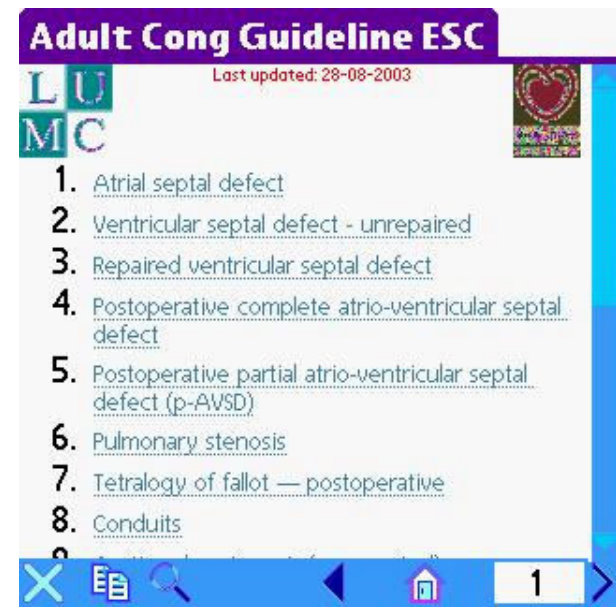

Figure 1. Example of the guidelines menu layout as created in iSilo. 
The guidelines are created (in HTML format) with Microsoft Frontpage and then converted into iSilo ${ }^{\mathrm{TM}}$ format or TomeRaider ${ }^{\mathrm{TM}}$ format. Isilo ${ }^{\mathrm{TM}}$ is a web browser application that runs on the Palm ${ }^{\mathrm{TM}}$ Operating System; there is also a version available for the Pocket PC. The same iSilo ${ }^{\mathrm{TM}}$ document can be used on a Palm ${ }^{\mathrm{TM}}$ PDA or Pocket-PC PDA. An example of a guideline in iSilo ${ }^{\mathrm{TM}}$ format on a Palm ${ }^{\mathrm{TM}} \mathrm{PDA}$ is shown in figure 1.

In addition to the guidelines in PDA format, the project group has selected a number of PDA applications that will be of use for all centers, such as risk-prediction software [2], medical calculators and drug reference software.

\subsection{Website}

A new, dedicated website has been established for this project (www.icin-ict.nl). All available PDA clinical guidelines and medical applications have been made available for download on the ICIN-ict website (see Figure 2).

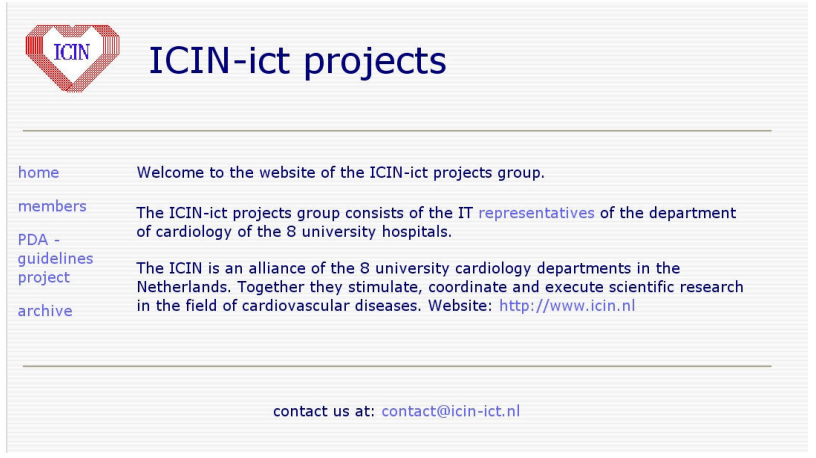

Figure 2. Screenshot of the ICIN-ict projects group website.

\section{Results}

The project has officially started in summer 2004, and therefore we will only be able to present some preliminary results.

\subsection{ICIN-ict website}

The website has been started very recently. A number of the PDA documents that are in use in the various centers have been made available on the website both in iSilo $^{\mathrm{TM}}$ and in TomeRaider ${ }^{\mathrm{TM}}$ format (see Figure 3). Also, a number of PDA applications have been placed on the ICIN-ict website.

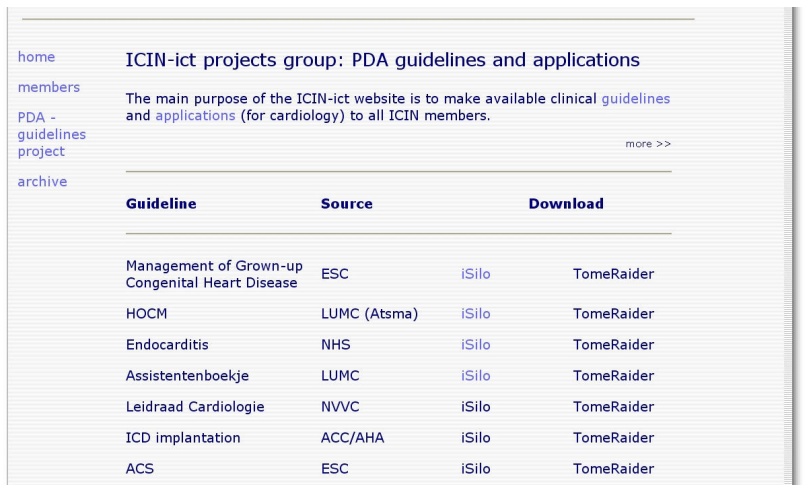

Figure 3. Screenshot of the Guidelines page on the ICINict website.

\subsection{Website implementation}

To allow all ICIN-ict project members to upload documents and adapt the ICIN-ict website, a Content Management System (CMS) (Mambo, http://www.mamboserver.com/) was installed on the website. With this open-source CMS system, authorized authors can easily make their own contributions to the website. The CMS system itself is administered by two ICIN-ict representatives.

The main purpose is to create access for the ICIN community to all PDA guidelines that have been developed in the ICIN centers. In addition, the website will also be used by the participants (ICT representatives of the ICIN centers) to share and exchange information in electronic format.

\subsection{Evaluation}

An important part of this project is to investigate if and how people use the PDA in the clinical decision making process. At specific moments it will be evaluated how often the PDA is used, and how satisfied the users are with the PDA for this specific purpose. Results from these local surveys then will be combined into a periodic report on the project, with recommendations for improvements and additional PDA documents and applications.

In one of the centers (the LUMC) the usage of the PDA is also regularly assessed. Normally, users synchronize the content of the PDA with the information on their desktop PC. In the LUMC, the local PDA documents and applications are available on a central network server. Dedicated software (XTND Connect) is used for central synchronization and administration. The PDA usage is assessed from the XTND Connect synchronization logs. The number of times users synchronize their PDA is thought to be representative for 
how much they use their PDA. Figure 4 shows a histogram of the number of synchronizations per user in a period of 2 months (40 working days). For this specific period, the median number of syncs was 13.5 , or one sync every 3 working days. From the histogram (based on data from the LUMC only) it can be clearly seen that there are a small number of users that synchronize their PDA very regularly (1-2 times a day), and that there is larger number of users that only synchronize infrequently.

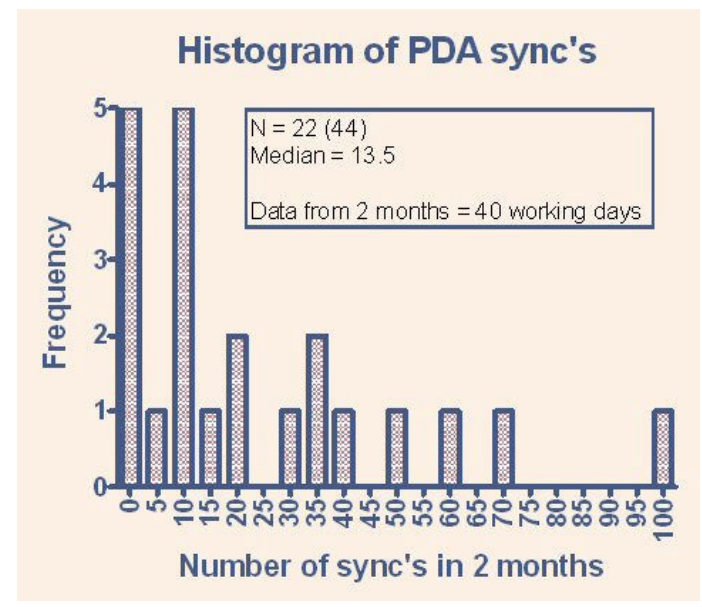

Figure 4. Histogram of the number of PDA syncs per user in a (specific) period of 2 months. Data from the LUMC only.

\section{Discussion and conclusions}

In many of the ICIN centers PDA's (Personal Digital Assistant) are used to support the cardiologist in the medical decision making process. In a number of these ICIN centers, PDA versions of relevant clinical guidelines are being developed. Also various medical PDA applications are evaluated and distributed locally. The purpose of this project is to give a boost to these local activities in the ICIN centers, and to make all these PDA guideline documents and applications available to all ICIN centers and other interested hospitals.

Preliminary results show that the ICIN-ict website is a useful reference site for PDA users in the ICIN centers. However, more documents and applications should be made available to really make it useful.
An editorial board has to be formed in order to assess the quality and correctness of the guideline documents and software. New guidelines, and software that has not been tested thoroughly yet should obtain a "preliminary" status. Also closer thoughts must be given to legal matters such as who owns the data, and who is responsible for its use.

An important part of this project is to investigate if and how people use the PDA in the clinical decision making process. At specific moments it will be evaluated how often the PDA is used, and how satisfied the users are with the PDA for this specific purpose. Results from these local surveys then will be combined into a periodic report on the project, with recommendations for improvements and additional PDA documents and applications, which will also be published on the website.

The results of a first survey (in the LUMC) are somewhat disappointing. Only a small number of users appear to synchronize their PDA regularly. However, this is not different from the experiences with the introduction of other new technologies. On the other hand, these results, and also the results of paper surveys, indicate that much effort needs to be put into these type of projects to make them useful for a large group of users.

\section{Acknowledgements}

This project is financially supported by a grant from Pfizer bv, The Netherlands.

\section{References}

[1] van der Velde ET, Atsma DE, Foeken H, Witteman TA, Schalij MJ, Van der Wall EE. Improvement in Quality of Care by Mobile Access to Always-up-to-Date Clinical Guidelines and Documentation of the Decision Process. Computers in Cardiology 2003;30:764-767.

[2] Becht AA, van der Velde ET, Atsma DE, Hoekstra WHGJ, van Dieren EB, Jonker LS. Implementing national guidelines on risk prediction and primary prevention of coronary heart disease in a cardiology information system. Computers in Cardiology 2002;29:389-392.

Address for correspondence

Enno T. van der Velde, $\mathrm{PhD}$

Leiden University Medical Center, C5-P

P.O. BOX 9600, 2300 RC Leiden University Medical Center The Netherlands

E-mail address: ETvanderVelde@lumc.nl 